\title{
Boron activation and diffusion in silicon and strained silicon-on-insulator by rapid thermal and flash lamp annealings
}

F. Lanzerath, D. Buca, H. Trinkaus, M. Goryll, S. Mantl, J. Knoch, U. Breuer, W. Skorupa, and B. Ghyselen

Citation: Journal of Applied Physics 104, 044908 (2008);

View online: https://doi.org/10.1063/1.2968462

View Table of Contents: http://aip.scitation.org/toc/jap/104/4

Published by the American Institute of Physics

\section{Articles you may be interested in}

Mechanisms of boron diffusion in silicon and germanium

Journal of Applied Physics 113, 031101 (2013); 10.1063/1.4763353

Diffusion of boron in silicon during post-implantation annealing Journal of Applied Physics 69, 2135 (1991); 10.1063/1.348740

Physics of strain effects in semiconductors and metal-oxide-semiconductor field-effect transistors Journal of Applied Physics 101, 104503 (2007); 10.1063/1.2730561

Transient enhanced diffusion of boron in $\mathrm{Si}$

Journal of Applied Physics 91, 8919 (2002); 10.1063/1.1471941

Rapid thermal annealing of arsenic and boron-implanted silicon

Applied Physics Letters 43, 957 (1983); 10.1063/1.94200

Rapid annealing and the anomalous diffusion of ion implanted boron into silicon Applied Physics Letters 50, 416 (1987); 10.1063/1.98160

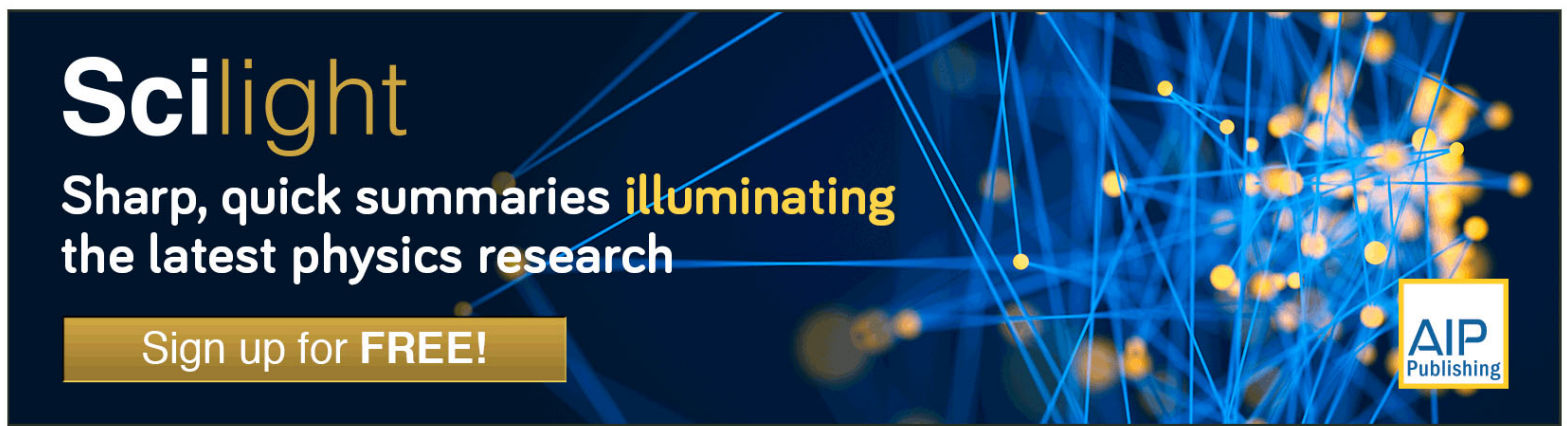




\title{
Boron activation and diffusion in silicon and strained silicon-on-insulator by rapid thermal and flash lamp annealings
}

\author{
F. Lanzerath, ${ }^{1}$ D. Buca, ${ }^{1, a)}$ H. Trinkaus, ${ }^{1}$ M. Goryll, ${ }^{1}$ S. Mantl, ${ }^{1}$ J. Knoch, ${ }^{2}$ U. Breuer, ${ }^{3}$ \\ W. Skorupa, ${ }^{4}$ and B. Ghyselen ${ }^{5}$ \\ ${ }^{1}$ Institute of Bio- and Nanosystems (IBN1-IT), Forschungszentrum Jülich, 52425 Jülich, Germany \\ ${ }^{2}$ Zurich Research Laboratory, IBM Research GmbH, 8803 Rüschlikon, Switzerland \\ ${ }^{3}$ Central Division of Analytical Chemistry (ZCH), Forschungszentrum Jülich, 52425 Jülich, Germany \\ ${ }^{4}$ Institute of Ion Beam Physics and Materials Research, Forschungszentrum Dresden-Rossendorf, 01314 \\ Dresden, Germany \\ ${ }^{5}$ SOITEC, Parc Technologique des Fontaines, 38190 Bernin, France
}

(Received 2 May 2008; accepted 13 June 2008; published online 28 August 2008)

\begin{abstract}
We present experimental results on the activation and diffusion behaviors of boron in silicon-on-insulator and strained silicon-on-insulator using standard rapid thermal processing treatments as well as flash lamp annealing. After boron implantation at different doses and at a low energy of $1 \mathrm{keV}$, samples were annealed to activate the dopants, and secondary ion mass spectrometry and Hall measurements were carried out to determine boron diffusion and the amount of activated dopants, respectively. In contrast to rapid thermal annealing, flash lamp annealing enables the activation without significant diffusion of dopants. In addition, we investigated the effect of coating the samples with antireflection layers to increase the absorbed energy during flash annealing. As a result, the activation was increased significantly to values comparable with the activation obtained with standard annealing. Furthermore, the relation between the observed boron diffusion and activation as a function of the implantation and annealing parameters is discussed in terms of the kinetics of the defects involved in these processes. (C) 2008 American Institute of Physics. [DOI: 10.1063/1.2968462]
\end{abstract}

\section{INTRODUCTION}

Silicon-on-insulator (SOI) will likely replace bulk silicon as complementary metal-oxide-semiconductor (CMOS) devices approach the $22 \mathrm{~nm}$ technology node. ${ }^{1}$ This is due to the performance and fabrication benefits of SOI, including lower power consumption, reduction of short channel effects, higher speed, and diminished latchup effects. ${ }^{2}$ Moreover, strained SOI (sSOI) has recently attracted a great deal of attention since it enables further performance improvements due to higher carrier mobility without device scaling while requiring only small changes in the device processing flow compared to SOI. ${ }^{3,4}$ When scaling down the dimensions of metal-oxide-semiconductor field effect transistor devices, ultrashallow contacts and extremely abrupt junctions between the source/drain electrodes and the channel are needed in order to suppress short channel effects. At the same time, the source/drain contacts must be highly doped to keep parasitic resistances as small as possible. Thus, the formation of appropriate source/drain electrodes becomes an increasingly difficult part of the device fabrication, and the performance and scaling advantages of SOI and, in particular, sSOI can only be exploited if such source/drain contacts can be realized.

Thermal annealing techniques such as rapid thermal processing (RTP) and spike RTP have been adequate for present CMOS technology but will become unsuitable for future devices as the dimensions decrease further. The reason is that

${ }^{a)}$ Electronic mail: d.m.buca@fz-juelich.de. the heating and cooling rates in RTP are limited to a few tens of ${ }^{\circ} \mathrm{C} / \mathrm{s}$, yielding an unacceptable broadening of the junction profiles and an increase in the junction depth of the source/ drain contacts. Particularly the formation of $p$-type contacts is troublesome since boron diffuses rapidly via self-interstitials. ${ }^{5}$ In addition, the source/drain formation in sSOI is difficult due to the lower solubility and the higher diffusion coefficient of boron induced by the tensile strain. ${ }^{6,7}$ On the other hand it was recently shown that flash lamp annealing (FLA) allows us to obtain ultrashallow $p$-junctions with high activation in bulk silicon. ${ }^{8-10}$ Due to annealing times in the millisecond range, only the surface region of the samples is heated such that the bulk remains at a significantly lower temperature, essentially representing a heat sink. ${ }^{8}$ In fact, FLA heating and cooling rates are higher by four orders of magnitude than RTP.

In the present paper we investigate the activation and diffusion behaviors of boron in SOI and sSOI by comparing RTP with FLA. The aim of the present study is to determine thermal processing conditions leading to high activation and, at the same time, very small diffusion of boron in SOI and sSOI. To this end, Hall measurements that allow us to determine the amount of dopant activation are carried out in combination with secondary-ion-mass-spectrometry (SIMS) measurements for a number of different dopant implantation and annealing conditions. In addition, further improvement of the FLA technique has been achieved by investigating the absorption properties of SOI/sSOI and the use of antireflection coatings in order to increase the energy deposited during a single flash light pulse. 


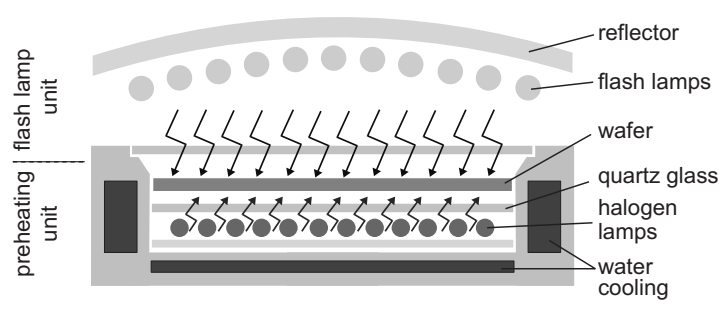

FIG. 1. Schematic drawing of the FLA system.

\section{SAMPLE PREPARATION}

Device grade SOI and sSOI samples $\left(2 \times 2 \mathrm{~cm}^{2}\right)$ were implanted with boron at an energy of $1 \mathrm{keV}$ and nominal doses of $5 \times 10^{14}, 1 \times 10^{15}$, and $3 \times 10^{15}$ ions $/ \mathrm{cm}^{2}$, respectively. The SOI consisted of a $70 \mathrm{~nm}$ silicon top layer and a $400 \mathrm{~nm}$ thick buried oxide (BOX), whereas the sSOI had a top layer of $70 \mathrm{~nm}$ tensely biaxial strained silicon and only a $140 \mathrm{~nm}$ thick BOX. The strained layer was created with the graded buffer method-a technique increasing the lattice parameter stepwise, ${ }^{11}$ in our case up to $0.84 \%$, corresponding to a negative pressure of about $1 \mathrm{GPa}$.

After implantation, dopant activation was carried out by either standard RTP or FLA. The RTP anneals were performed in nitrogen atmosphere using a STEAG SHS-100 tool. Four different temperature and time conditions were chosen, namely, (i) spike annealing at $1000{ }^{\circ} \mathrm{C}$, (ii) $5 \mathrm{~s}$ annealing at $1000{ }^{\circ} \mathrm{C}$, (iii) $20 \mathrm{~s}$ annealing at $1000{ }^{\circ} \mathrm{C}$, and (iv) spike annealing at $1050{ }^{\circ} \mathrm{C}$; for all cases, the same heating and cooling rates of about $25^{\circ} \mathrm{C} / \mathrm{s}$ were applied. The thermal activation with flash lamps was carried out at the FLA facility of the Forschungszentrum Dresden-Rossendorf. The FLA system consists of a process chamber, an upper bank of xenon lamps, a wafer holder, a lower bank of halogen lamps, and two sets of aluminum reflectors behind each bank of lamps, as depicted in Fig. 1. By discharging a capacitor/ inductor unit, an intense pulse of light is generated. To control the flash light intensity, the charge stored in the capacitors is varied. Since it is not possible to accurately determine either the energy input or the temperature of the samples during the process, the total energy stored in the capacitors is used as a parameter of FLA because the energy can be controlled, precisely guaranteeing a well-defined setup. In our experiments the capacitors were charged with energies in the range of 45.9-88.6 kJ, which is the maximum energy load of the system. Adjusting the inductor unit, it is possible to determine the length of the light pulse. In the present case, a minimum of $3 \mathrm{~ms}$ was chosen in all following experiments. In order to increase the maximum temperature and to minimize the thermally induced stress of the SOI/sSOI wafers, the halogen lamps were used to preheat the samples to a temperature of $650{ }^{\circ} \mathrm{C}$. The chosen pulse duration and preheating temperature ensure optimum conditions in terms of dopant diffusion and activation as shown by Skorupa et al. ${ }^{12}$ for bulk silicon. The whole process of preheating and FLA took place in an inert ambient of argon. More details of the FLA system are published elsewhere. ${ }^{13}$

A major difference between bulk silicon and SOI/sSOI is the layered structure with several dielectric boundaries between silicon and $\mathrm{SiO}_{2}$. Consequently, the energy deposited in the top silicon layer used to activate the dopants can be expected to be significantly different in SOI/sSOI and in bulk silicon since a large fraction of the light during FLA is either reflected back or transmitted to the substrate.

It will be discussed below that the amount of energy that is reflected back can be substantially decreased by depositing an antireflection layer on the sample surface, thereby also increasing the amount of energy deposited in the top silicon layer. In the present case an approximately $240 \mathrm{~nm}$ thick $\mathrm{SiO}_{2}$ layer was used. It was deposited employing plasmaenhanced chemical vapor deposition (PECVD). To avoid any dopant diffusion, the deposition temperature of the silicon dioxide layer was kept at $300{ }^{\circ} \mathrm{C}$. After the thermal treatment by FLA, the coating was removed with hydrofluoric acid (HF).

Electrical measurements as well as SIMS analysis were carried out in order to characterize all samples regarding the activation and diffusion of dopants. For the electrical characterization of the samples, Hall bar structures were fabricated employing standard optical lithography and reactive ion etching with a sulfur hexafluoride/argon plasma to define Hall bar shaped mesas. Afterward, aluminum contacts were patterned using a lift-off process. Prior to the deposition of the aluminum, the native oxide was removed with HF. Hall measurements were carried out at room temperature to obtain the total sheet carrier density and hence the amount of electrically active dopants. In addition, the Hall mobility was measured. To investigate the dopant diffusion, SIMS was employed before and after the thermal treatment of the samples. A low-energy $\mathrm{O}_{2}^{+}$ion beam of $2 \mathrm{keV}$ was used for the SIMS analysis in order to achieve a good depth resolution.

An important issue in the case of the sSOI is the impact of the implantation/activation procedure on the strain level within the top silicon layer. We have therefore measured the strain level in the sSOI samples by Raman spectroscopy before and after the implantation/FLA steps. We did not find any impact of the FLA processing on the strain level in the sSOI samples.

\section{RESULTS AND DISCUSSION}

\section{A. Dopant activation using RTP}

RTP samples were fabricated as references that can be compared with the samples treated with FLA. All processing steps apart from annealing were kept identical to facilitate a fair comparison. Figure 2 shows the depth profiles of 1 $\times 10^{15}$ ions $/ \mathrm{cm}^{2}$ implanted SOI samples.

It can be seen that diffusion takes place in all cases. Even for the lowest thermal budget $\left(1000{ }^{\circ} \mathrm{C}\right.$, spike), a junction broadening (at $3 \times 10^{18} \mathrm{~cm}^{-3}$ ) of more than $15 \mathrm{~nm}$ occurs. For higher thermal budgets the diffusion increases, and boron almost reaches the BOX at $70 \mathrm{~nm}$. For the longest activation time of $20 \mathrm{~s}$, the diffusion is only stopped due to the BOX, and a high concentration of $2 \times 10^{19} \mathrm{~cm}^{-3}$ is found at $70 \mathrm{~nm}$. All RTP-annealed sSOI samples show the same dependence of the diffusion behavior on the processing conditions, with a somewhat higher diffusion compared to the unstrained SOI samples. This observation is consistent with 


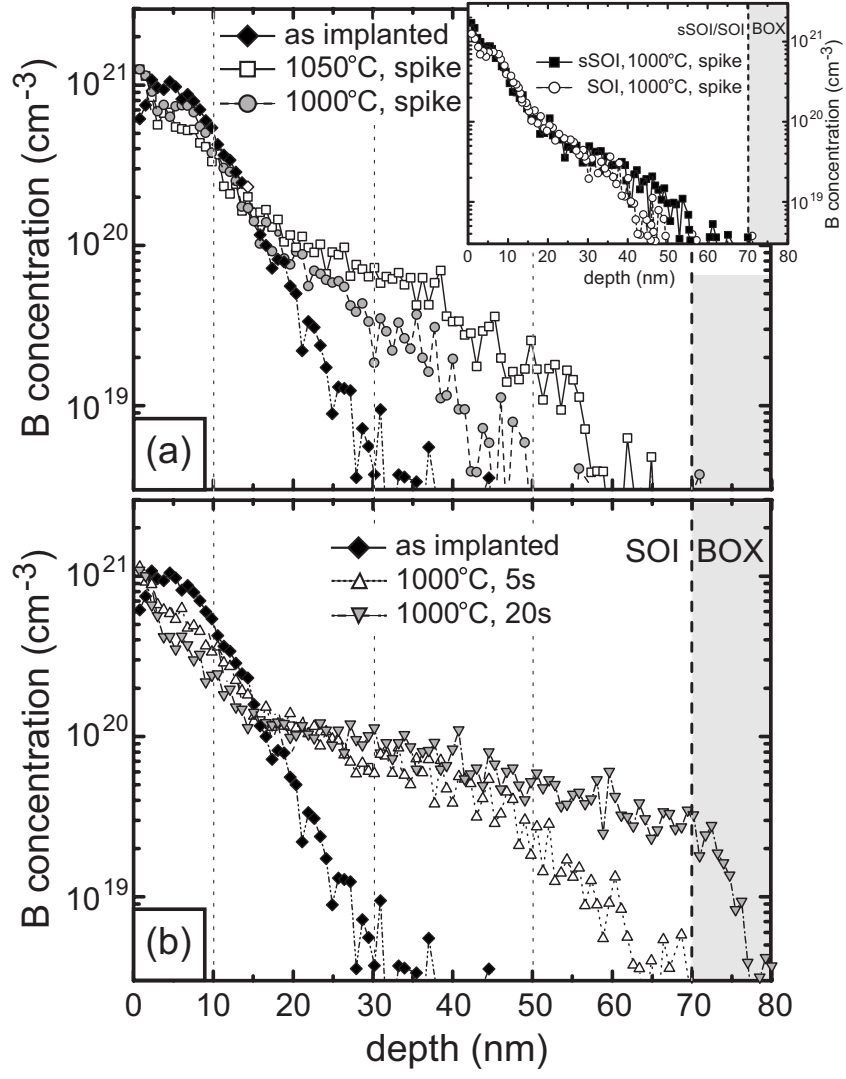

FIG. 2. SIMS profiles of $1 \times 10^{15}$ ions $/ \mathrm{cm}^{2}$ boron implanted RTP treated SOI samples for (a) spike annealings at 1000 and $1050^{\circ} \mathrm{C}$. (b) shows RTP samples annealed at $1000{ }^{\circ} \mathrm{C}$ for 5 and $20 \mathrm{~s}$.

the findings of Ref. 6. As an example, the inset in Fig. 2(a) shows a comparison of spike annealed SOI and sSOI samples.

Hall measurements show that the dopant activation increases with larger annealing times and temperatures. For the $1 \times 10^{15} \mathrm{~cm}^{-2}$ implanted samples, the activation increases from $4.70 \times 10^{14} \mathrm{~cm}^{-2}$ (spike) to $6.68 \times 10^{14} \mathrm{~cm}^{-2}(5 \mathrm{~s})$ to $7.46 \times 10^{14} \mathrm{~cm}^{-2}(20 \mathrm{~s})$ at $1000{ }^{\circ} \mathrm{C}$, corresponding to $47 \%$, $66.8 \%$, and $74.6 \%$, respectively.The best trade-off between diffusion and activation is obtained with spike annealing at $1050{ }^{\circ} \mathrm{C}$, which yields an activation of $6.87 \times 10^{14} \mathrm{~cm}^{-2}$ (Fig. 3).

The general dependence of activation on implantation dose and time may be summarized as follows: The increase in activation at a given dose and temperature with increasing
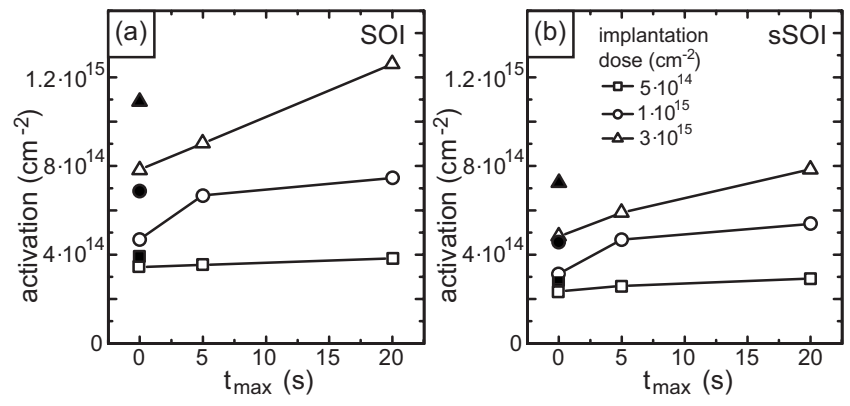

FIG. 3. Boron activation of (a) SOI and (b) sSOI for all implantation doses using RTP activation at $1000{ }^{\circ} \mathrm{C}$ (hollow symbols) and $1050{ }^{\circ} \mathrm{C}$ (solid symbols).

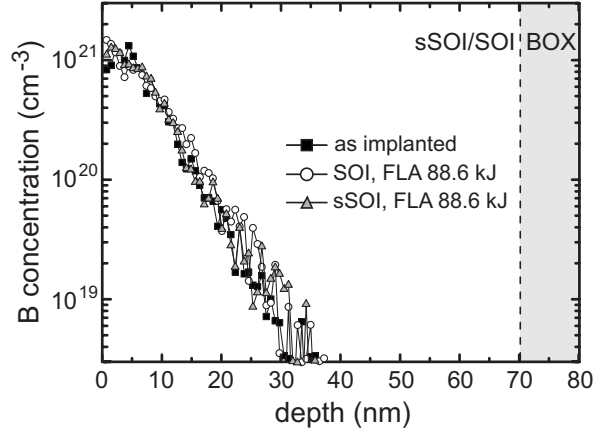

FIG. 4. SIMS profiles of FLA treated SOI and sSOI samples at the highest capacitor load of $88.6 \mathrm{~kJ}$ and an implantation dose of $1 \times 10^{15} \mathrm{~cm}^{-2}$.

annealing time accelerates with increasing dose. At a given RTP temperature and time, the activation increases sublinearly with increasing dose; i.e., the activated fraction of implanted B decreases with increasing dose, almost independent of RTP temperature and time. In the sSOI samples, the trends are similar, but the activation is reduced by about $30 \%$. The relative reduction increases somewhat with increasing dose and annealing time.

\section{B. Dopant activation using FLA}

Figure 4 shows typical SIMS profiles of selected FLA samples. The displayed profiles belong to $1 \times 10^{15} \mathrm{~cm}^{-2} \mathrm{im}$ planted SOI and sSOI samples, which were annealed with the maximum energy load of the Rossendorf FLA facility. The result of this SIMS analysis is that within the precision of measurements, no significant boron diffusion is found. Both FLA profiles lie close to the profile of the unannealed as-implanted reference sample. It should be mentioned that we did not find any substantial dopant redistribution in all flash lamp annealed samples that were studied with SIMS. Compared to RTP, the FLA technique exhibits therefore a significant advantage regarding dopant diffusion.

All flash lamp annealed samples were also electrically analyzed in detail. The dopant activation for various samples is plotted in Fig. 5. Two different FLA series ranging from 45.9 to $67.0 \mathrm{~kJ}$ and from 67.0 to $88.6 \mathrm{~kJ}$ were carried out. Although the curves show the same trend, the absolute value of the activation at $67.0 \mathrm{~kJ}$ differs in almost every case. The reason for the difference is most likely due to the limited reproducibility of the annealing parameters in our system.
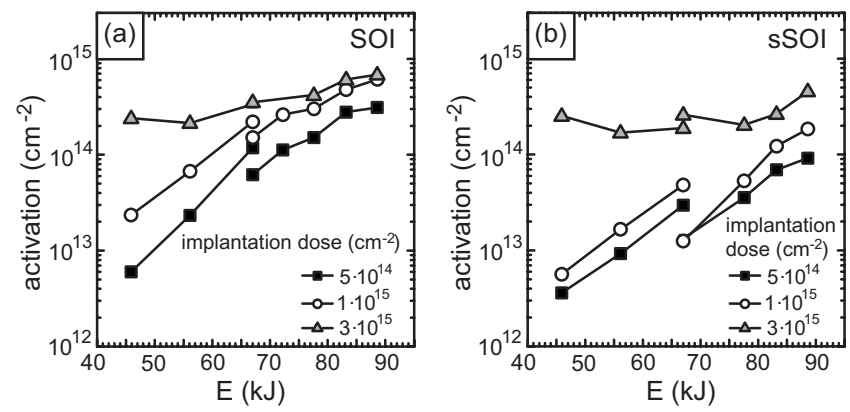

FIG. 5. Boron activation of (a) SOI and (b) sSOI for all implantation doses and all FLA activation energies. 
TABLE I. Electrical characteristics of $1 \times 10^{15} \mathrm{~cm}^{-2}$ implanted RTP and FLA samples.

\begin{tabular}{ccccc}
\hline \hline Annealing & $R(\Omega / \mathrm{sq})$ & \multicolumn{3}{c}{ Activation $\left(10^{14} \mathrm{~cm}^{-2}\right)$} \\
& SOI & sSOI & SOI & sSOI \\
\hline Spike at $1000^{\circ} \mathrm{C}$ & 339 & 382 & 4.70 & 3.14 \\
Spike at $1050^{\circ} \mathrm{C}$ & 223 & 270 & 6.87 & 4.62 \\
FLA with $88.6 \mathrm{~kJ}$ & 306 & 727 & 6.11 & 1.84 \\
\hline \hline
\end{tabular}

In Fig. 5 it can be seen that the activation increases with higher FLA energies; the steeper the slope of the curves, the lower the implantation dose. Because of this, the dose dependence of activation decreases with increasing FLA energy and becomes weak and similar to that found in RTP at high energies. At the lowest energy of $45.9 \mathrm{~kJ}$, the dose dependence of activation is strikingly superlinear; i.e., the fraction of activated B increases significantly with increasing dose. For instance, for implantation of $1 \times 10^{15} \mathrm{~cm}^{-2}$ into SOI/ sSOI, the fraction increases from $2.4 \% / 0.6 \%$ to $61 \% / 18 \%$, respectively. The ratio of the activations of the SOI and the sSOI samples reaches values up to 6, i.e., significantly larger values than those in RTP except for the highest dose of 3 $\times 10^{15} \mathrm{~cm}^{-2}$. As as result, in the case of smaller implantation doses, an adequate dopant activation in SOI/sSOI can only be achieved with high energies, whereas low energies result in insufficient activation. The sheet resistance measurement confirms this result: to obtain low sheet resistances, a high FLA energy is necessary. In comparison with the RTP samples, the $88.6 \mathrm{~kJ}$ flash lamp annealed SOI samples exhibit similar activation and sheet resistances, as listed in Table I. However, in the case of sSOI, annealing with flash lamp yields activation levels and sheet resistances that are considerably lower than those of the RTP treated sSOI samples as well as the SOI samples. A possible reason for this is a higher reflectivity of sSOI compared to SOI, which would have a minor impact on the activation using RTP but a significant effect when annealed using FLA. In the next section, we will therefore study the optical properties of the $\mathrm{SOI} / \mathrm{sSOI}$ multilayer system. As it turns out, only a small fraction of the overall FLA energy is deposited in the top silicon layer and can be used for the activation. A possible solution to increase the activation level in sSOI is to employ an antireflection coating, which will be discussed in detail below.

\section{Reflection and absorption in SOI/sSOI}

The activation levels obtained in our FLA experiments in SOI and sSOI can be achieved in bulk $\mathrm{Si}$ at much lower FLA energies. $^{12}$ This again, shows the importance of the layer structure of the samples under investigation. Therefore, we calculate the amount of reflected and transmitted energy as well as the energy deposited within the top silicon layer in the cases of SOI and sSOI. Using the matrix method for multiple reflections and transmissions at all interfaces, ${ }^{14}$ we calculate the reflection and transmission at each interface and the absorption in each layer as functions of the wavelength of the incident light.

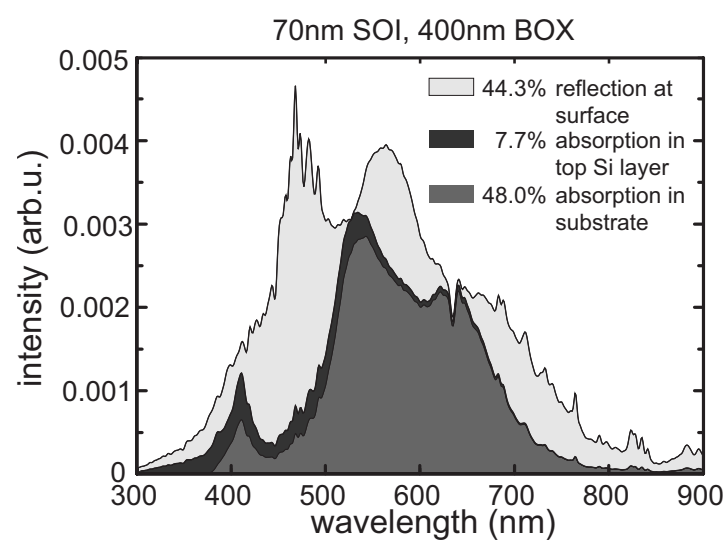

FIG. 6. Optical spectrum of the reflected light and the fractions absorbed within the $70 \mathrm{~nm}$ SOI layer and the substrate.

The simulation result for $70 \mathrm{~nm}$ SOI sample is illustrated in Fig. 6. The envelope represents the flash lamp spectrum, and the areas underneath the envelope represent the energy fraction reflected at the surface, the fraction absorbed in the substrate, and the fraction absorbed in the top layer. In the case of SOI $44 \%$ of the flash lamp energy is reflected at the surface, whereas sSOI exhibits a reflectivity of $48 \%$. The deviation mainly results from the thickness of the BOX layer, which is $400 \mathrm{~nm}$ for SOI and only $140 \mathrm{~nm}$ in the case of sSOI, determining the optical characteristics of the SOI/ sSOI samples.

For SOI and sSOI the transmitted energy through the sample surface can be subdivided into the part absorbed in the top (strained) silicon layer and the part that is transmitted further into the silicon substrate and absorbed there. Our calculations yield $7.7 \%$ absorption in the top silicon layer in the case of SOI and $7.5 \%$ for sSOI; the absorption in the substrate is $48 \%$ for SOI and $44 \%$ for sSOI (see above). Since there is only a small difference in the amount of absorbed energy, it appears that the generically lower activation in sSOI is the relevant factor for the lower boron activation in sSOI compared to SOI.

In order to improve the absorption characteristics, we computed the optical properties of SOI/sSOI with an additional single cap layer of silicon dioxide serving as antireflection coating. To obtain the optimum thickness for the antireflection layer, the calculations were performed for oxide thicknesses between $1 \mathrm{~nm}$ and $1 \mu \mathrm{m}$. A good trade-off between minimizing the reflection and maximizing the absorption in the top layer of both SOI and sSOI is found at $\sim 240 \mathrm{~nm}$, which allows a better experimental comparison. The results of the simulation are summarized in Table II. In both cases, the antireflection coating gives rise to an increase in the amount of absorbed energy of approximately $20 \%$. In Fig. 5 it is apparent that this should result in a significant increase in the activation level, particularly in the case of lower implantation doses. If an antireflection coating is applied, a strong increase in the activation and a decrease in the sheet resistance can be observed experimentally, which will be discussed in the following. 
TABLE II. Calculated influence of the antireflection layer $\left(240 \mathrm{~nm} \mathrm{SiO}_{2}\right)$ on the optical characteristics.

\begin{tabular}{lccc}
\hline \hline \multicolumn{1}{c}{ Values in $\%$} & $\begin{array}{c}\text { Reflection } \\
\text { at the surface }\end{array}$ & $\begin{array}{c}\text { Absorption } \\
\text { in the top layer }\end{array}$ & $\begin{array}{c}\text { Absorption } \\
\text { in the substrate }\end{array}$ \\
\hline SOI & 44.3 & 7.7 & 48.0 \\
SOI with cap & 38.2 & 9.3 & 52.5 \\
Difference & -6.1 & 1.6 & 4.5 \\
sSOI & 48.4 & 7.5 & 44.1 \\
sSOI with cap & 42.5 & 8.9 & 48.6 \\
Difference & -5.9 & 1.4 & 4.5 \\
\hline \hline
\end{tabular}

\section{Activation using an antireflection layer}

The deposition of the silicon dioxide cap layer was performed in a PECVD tool at a low temperature of only $300{ }^{\circ} \mathrm{C}$ in order to avoid any dopant redistribution caused by this process. The thickness of the antireflection coating was determined with ellipsometry to be approximately $240 \mathrm{~nm}$, as designated by our simulation. We applied the antireflection layer just before the step of FLA and removed it with $\mathrm{HF}$ afterward. All other processing steps were carried out equal to the steps of the samples without coating, assuring a good comparability.

In the subsequent FLA an energy of $83.2 \mathrm{~kJ}$ was used, slightly lower than the maximum in order to prevent the samples from being damaged by thermal stress. The results of the electrical characterization are compiled in Table III. As can be seen in this table, a higher activation and a lower sheet resistance are obtained in samples with antireflection coating. The sheet resistance for both SOI and sSOI drops by more than a factor of 2 ; the relative reduction for each sample is approximately the same. These results show the effectiveness and usefulness of an antireflection layer in increasing the amount of energy deposited when annealing SOI/sSOI. Consequently, FLA yields superior results for dopant activation since it enables activation levels comparable to standard RTP virtually free of dopant diffusion. However, a closer look at the electrical characteristics also reveals different behaviors of SOI and sSOI. In the case of SOI the use of the antireflection layer results in a 100\%increase in boron activation and a slight increase in carrier mobility. The sSOI sample, on the other hand, shows an even higher increase in activation, more than $200 \%$, but exhibits a

TABLE III. Influence of the antireflection layer on the electrical characteristics of SOI/sSOI samples implanted at $1 \times 10^{15} \mathrm{~cm}^{-2}$ and annealed at 83.2 kJ FLA.

\begin{tabular}{lccc}
\hline \hline & $\begin{array}{c}\text { Sheet resistance } \\
(\Omega / \mathrm{sq})\end{array}$ & $\begin{array}{c}\text { Activation } \\
\left(10^{14} / \mathrm{cm}^{2}\right)\end{array}$ & $\begin{array}{r}\text { Mobility } \\
\left(\mathrm{V} \mathrm{s} / \mathrm{cm}^{2}\right)\end{array}$ \\
\hline SOI & 730 & 2.70 & 33.38 \\
SOI with cap & 303 & 5.55 & 37.08 \\
Absolute change & -426 & 2.84 & 3.70 \\
Relative change $(\%)$ & -58.4 & 105.2 & 11.09 \\
SOI & 1538 & 0.80 & 51.59 \\
SOI with cap & 599 & 2.49 & 42.03 \\
Absolute change & -939 & 1.69 & -9.56 \\
Relative change $(\%)$ & -61.1 & 212.2 & -18.53 \\
\hline \hline
\end{tabular}

decrease in mobility. A possible explanation for this mobility drop in sSOI is the high boron activation itself, which leads to a substantial increase in ionized impurity scattering.

In order to obtain high activation and small sheet resistances, it is vital to deposit sufficient amounts of energy. At the same time, the induced thermal stress must be kept small enough to avoid damaging the SOI/sSOI samples. This can be accomplished by optimizing the ratio of the energy absorbed in the top layer and the substrate and by keeping the overall thermal budget as low as possible. With our experiments a first step is done toward employing antireflection layers in FLA of SOI/sSOI samples, whereby an increase in the deposited energy into their top silicon layers was achieved.

\section{E. Kinetics of dopant activation}

In the following, we make an attempt to rationalize our experimental results on boron diffusion and activation as functions of the implantation parameters and, particularly, of the annealing parameters differing substantially between RTP and FLA. We start by discussing the SIMS profiles shown in Figs. 2 and 4 for the samples treated by RTP and FLA, respectively. Details will be published elsewhere.

The most striking feature of the profiles shown in Fig. 2 is their unusual two-limb shape consisting of a relatively stable hump and a tail spreading substantially with increasing temperature and time. With this shape, the profiles differ qualitatively from typical diffusion profiles. We attribute this feature to a qualitative difference between the defect structures near the surface (S region) and away from it (bulk region) approached after annihilation of most of the Frenkel pairs produced upon ion implantation. Close to the surface, which represents a sink, a source for self-interstitial atoms (SIAs), and a source for vacancies, a state close to global thermal equilibrium reached after long annealing may be assumed to become established in a limited but extending region in which the majority of boron atoms are on substitutional sites. Using for this region the values of $7.87 \exp \left(3.75 \mathrm{eV} / k_{B} \mathrm{~T}\right) \mathrm{cm}^{2} / \mathrm{s}$ reported for the preexponential factor and the activation energy of $\mathrm{B}$ diffusion in $\mathrm{Si}^{15}$ we estimate about $5 \mathrm{~nm}$ profile broadening during $10 \mathrm{~s}$ RTA at $1000{ }^{\circ} \mathrm{C}$, which is reached by 3 ms FLA only close to the melting temperature of silicon. Thus, in both types of annealing, the boron profiles would broaden by less than $50 \%$, which contrasts drastically with the wide spreading of the profile tails, as shown in Fig. 2.

The difference between bulk and $\mathrm{S}$ regions may be related to differences either in their B concentration levels or in their distance to the surface, i.e., to the sources and sinks of self-defects. In the following discussion, the later possibility will be considered in some detail. In fact, in the bulk region, the transient local equilibrium reached after Frenkel pair annihilation is characterized by the lack of sufficiently close SIA sinks and vacancy sources. In particular, in this region, the concentration of vacancies surviving annihilation seems to be too low to provide a sufficient number of substitutional sites for the implanted boron atoms, except perhaps close to 


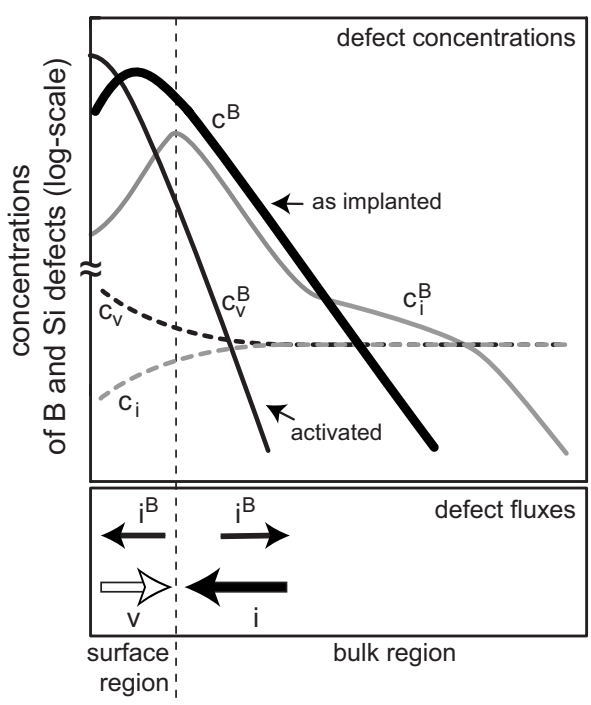

FIG. 7. Schematic illustration of the evolution of concentrations and fluxes of interstitial and substitutional B atoms, $i^{\mathrm{B}}$ and $v^{\mathrm{B}}$, and of interstitial and vacancy self-defects, $i$ and $v$, in the surface near the (S) and the bulk region during RTA (thin lines) following B implantation to a B concentration $v^{\mathrm{B}}$ (black, fat line).

the interface to the BOX layer, which may be expected to act as a vacancy source and will certainly be a good SIA sink.

The importance of the distance to sinks and sources of self-defects for the behavior of B atoms is related to the fact that ion implantation is necessarily connected with a local excess concentration of interstitial atoms, i.e., with a difference between the total concentration of B-interstitial and SIA, $c_{i}^{\mathrm{B}}+c_{i}$, on one hand, and the concentration of vacancies, $c_{v}$, on the other hand, defined by the local concentration of implanted ions, $c^{\mathrm{B}}=c_{i}^{\mathrm{B}}+c_{i}-c_{v}$. We think that, in the bulk region, after annihilation of implantation induced SIAs and vacancies, a local thermal Frenkel pair equilibrium is reached, where $c_{i} \approx c_{v}$ means $c_{i}^{\mathrm{B}} \approx c^{\mathrm{B}}$. Since, as generally agreed upon, the formation energy of SIAs is larger than that of vacancies, such a local equilibrium will imply a SIA supersaturation and a vacancy subsaturation with reference to the global equilibrium concentrations $c_{i, v}^{\mathrm{eq}}$, as established in the $\mathrm{S}$ region. This is the crucial difference between the two regions: In $\mathrm{S}$, where sufficient vacancies are available, the majority of B atoms are on substitutional sites and are virtually immobile there (like clusters of B defects or of B and self-defects ${ }^{16}$ ); in the bulk region, with $c_{i} \approx c_{v}$, the majority of $\mathrm{B}$ atoms are on interstitial sites, $c_{i}^{\mathrm{B}} \approx c^{\mathrm{B}}$, over which they perform fast diffusion (see Fig. 7). It is emphasized here that the interstitial $\mathrm{B}$ atoms would be electrically inactive but would represent scattering centers for electrons and holes (like B clusters).

We have analyzed the evolution of the tails of the B profiles to derive estimates for the fast B diffusion characteristics (assumed to be of interstitial or interstitialcy type [Ref. 5]). Considering spike RTP together with 5 and $20 \mathrm{~s} \mathrm{RTP}$ at $1000{ }^{\circ} \mathrm{C}$, we have estimated the diffusion coefficient in SOI at that temperature as $D^{\mathrm{B}} \approx 2 \times 10^{-13} \mathrm{~cm}^{2} / \mathrm{s}$. A comparison of spike RTPs at 1050 and $1000{ }^{\circ} \mathrm{C}$ yields an estimate of the activation energy of $2.2 \mathrm{eV}$. Note that the profile broadening around B layers containing B-self-interstitial clusters ${ }^{17}$ (BICs) is roughly consistent with our estimate of the fast B diffusion coefficient.

We can apply our results for fast B diffusion under the RTP conditions to $3 \mathrm{~ms}$ FLA. Assuming that the maximum energy of $88.6 \mathrm{~kJ}$ generates a temperature close to the melting point of $\mathrm{Si}$, we estimate profile shifts of the order of 1 $\mathrm{nm}$, consistent with the small shifts close to the resolution limit shown in Fig. 4.

For boron activation, two conditions must be fulfilled: (i) The supply of vacancies or the removal of SIAs from and to the surface (or the interface to the BOX layer) during the available time must be sufficient to provide the required substitutional sites for a substantial fraction of the implanted B atoms (and to annihilate BICs), and (ii) thus the resulting high temperature solid solution of B atoms must be frozen-in sufficiently rapidly to avoid significant boron precipitation at low temperatures. According to the first condition, coupled diffusion fluxes of self-defects and B atoms in $\mathrm{S}$ and bulk regions must be considered in discussing $\mathrm{B}$ activation, as schematically shown in the lower part of Fig. 7.

Quantitatively, the first condition means that the integrated vacancy and/or SIA flux from the surface (interface) to the implanted layer and in the opposite direction must reach a level of the order of the implanted boron dose. Using the reported values for the vacancy contribution to self-diffusion, ${ }^{5}$ we have estimated integrated vacancy fluxes during RTP to be below, even though close to, the lowest implantation doses. In $3 \mathrm{~ms}$ FLA, however, such (almost sufficient) values are not reached at temperatures below $1350{ }^{\circ} \mathrm{C}$. Consequently, for both annealing modes, the vacancy supply during the available time is not sufficient to convert a substantial fraction of the implanted B atoms into a substitutional solid solution. The contribution of fluxes of (supersaturated) SIAs from the bulk to the surface or to the BOX interface must be included to obtain sufficiently high total integrated self-defect fluxes on the order of $10^{15} \mathrm{~cm}^{-2}$ after $10 \mathrm{~s}$ for our RTP conditions. In $3 \mathrm{~ms}$ FLA, temperatures above $1300{ }^{\circ} \mathrm{C}$ would be required to obtain a similar level. In this respect, FLA appears to be more critical than RTP. This conclusion provides a theoretical justification for our efforts to increase the energy input into the samples in the FLA case as much as possible, for instance, by coating the samples with an antireflection layer.

At first glance, the activation of a rapidly spreading dopant distribution by a slowly moving equilibration front seems to be impossible. However, upon formation of a substitutional solid solution in the surface region, the local boroninterstitial concentration is drastically reduced, resulting in transient boron-interstitial fluxes from the bulk to the $\mathrm{S}$ region and in a corresponding increase in the boron concentration there, as schematically shown in Fig. 7.

According to Figs. 2 and 4, the B density is above 5 $\times 10^{20} \mathrm{~cm}^{-3}$ in a substantial fraction of the profiles shown. The data for the solubility of boron in silicon ${ }^{18}$ indicate that B solid solution densities above this value are reached only at temperatures above $1200{ }^{\circ} \mathrm{C}$, i.e., in our case only during high-energy FLA but not during RTA. The relatively high maximum B activations reached by both RTP and FLA show 
that a substantial fraction of the implanted B atoms are maintained in a solid solution during RTA and FLA locally supersaturated with respect to the nominal thermodynamic solubility limit. In the absence of near nucleation centers, this is not surprising for the temperatures/times considered. The effective solubility that matters here, however, is that at the "kinetic freezing point" of the solid solution, which depends on the cooling rate. With orders of magnitude higher cooling rates, FLA is clearly superior to RTP in this respect.

The B solubility is generally considered to be reduced by tensile stress because of the smaller size of the B atom compared to $\mathrm{Si}$. The reduction is described by $c(p) / c(p=0)$ $=\exp \left(-p V^{*} / k_{B} T\right)$, where $p$ is the hydrostatic pressure $(-2 / 3$ of the plane tensile stress) and $V^{*}$ is the solution volume assumed to be the difference between the atomic volumes of $\mathrm{B}$ and $\mathrm{Si}$, which is about -0.6 of that of the $\mathrm{Si}$. For the plane strain of $0.84 \%$, corresponding to $p \approx 1 \mathrm{GPa}$, in our (100) sSOI samples, the reduction factor will be around 0.5 for RTP at $1000{ }^{\circ} \mathrm{C}$. The smaller difference between the RTP treated sSOI and SOI samples is due to the fact that only the peak(s) of the profiles are cut down to the level of the reduced solubility limit. The much larger difference between the activation in the FLA treated sSOI and SOI samples compared with the RTP treated samples is partially due to the reduced energy input in the sSOI samples due to a thinner BOX layer.

We conclude this section with the remark that the level of activation around $10^{15} \mathrm{~cm}^{-2}$ reached by RTP with substantial boron diffusion can be obtained by FLA with insignificant boron diffusion only at the highest FLA energy and by coating the sample with an antireflection layer. In this case, the temperature in FLA comes probably close to the melting point of silicon.

\section{CONCLUSION}

We have studied the thermal activation of ultrashallow boron implantations in SOI and sSOI by FLA treatment. Hall measurements showed a high activation yield in both SOI and sSOI, comparable to the samples treated with RTP. Dopant diffusion was investigated by SIMS, and no boron redistribution could be observed even at the highest FLA energies. The boron activation of sSOI was usually lower than that of
SOI, which is mainly due to the higher reflectivity of sSOI. The energy deposition into the top silicon layers of SOI and sSOI was significantly increased by antireflection coatings. Boron activation in boron implanted SOI and sSOI samples by RTP and FLA is discussed in terms of the coupled kinetics of fast and slowly diffusing B defects and Si self-defects. FLA is found to be a viable alternative to the currently used RTP method. Its advantage over RTP is the electrical activation of ultrashallow boron junctions without considerable dopant diffusion.

${ }^{1}$ H.-S. P. Wong, IBM J. Res. Dev. 46, 133 (2002).

${ }^{2}$ G. K. Celler and S. Cristoloveanu, J. Appl. Phys. 93, 4955 (2003).

${ }^{3}$ N. Mohta and S. E. Thompson, IEEE Circuits Devices Mag. 21, 18 (2005).

${ }^{4}$ S. E. Thompson, G. Sun, K. Wu, J. Lim, and T. Nishida, Tech. Dig. - Int. Electron Devices Meet. 2004, 221.

${ }^{5} \mathrm{H}$. Bracht, MRS Bull. 25, 22 (2000).

${ }^{6}$ N. R. Zangenberg, J. Fage-Pedersen, J. Lundsgaard Hansen, and A. Nylandsted Larsen, J. Appl. Phys. 94, 3883 (2003).

${ }^{7}$ J. Adey, R. Jones, and P. R. Briddon, J. Phys.: Condens. Matter 16, 9117 (2004).

${ }^{8}$ W. Skorupa, R. A. Yankov, T. Gebel, W. Anwand, and M. Voelskow, Advances in Electronic Manufacturing Technology, V-EMT 1, 23 (2004)

${ }^{9}$ K. Tsutsui, Y. Sasaki, C.-G. Jin, H. Sauddin, K. Majima, Y. Fukagawa, I. Aiba, H. Ito, B. Mizuno, K. Kakushima, P. Ahmet, and H. Iwai, 14th Internatational Conference on Advanced Thermal Processing of Semiconductor, 2006 (unpublished), p. 39.

${ }^{10}$ W. S. Yoo and K. Kang, Nucl. Instrum. Methods Phys. Res. B 237, 12 (2005).

${ }^{11}$ M. L. Lee, E. A. Fitzgerald, M. T. Bulsara, M. T. Currie, and A. Lochtefeld, J. Appl. Phys. 97, 011101 (2005).

${ }^{12}$ W. Skorupa, R. A. Yankov, W. Anwand, M. Voelskow, T. Gebel, D. F. Downey, and E. A. Arevalo, Mater. Sci. Eng., B 114-115, 358 (2004).

${ }^{13}$ J. Stoemenos, D. Pankin, M. Eickhoff, V. Heera, and W. Skorupa, J. Electrochem. Soc. 151, G136 (2004).

${ }^{14}$ M. Smith, R. A. McMahon, M. Voelskow, and W. Skorupa, J. Appl. Phys. 96, 4843 (2004).

${ }^{15}$ Y. M. Haddara, B. D. Folder, M. E. Law, and T. Buyuklimanli, Appl. Phys. Lett. 77, 1976 (2000).

${ }^{16}$ G. Bisognin, D. De Salvador, E. Napolitani, L. Aldegheri, M. Berti, A. Carnera, A. V. Drigo, S. Mirabella, E. Bruno, G. Impellizeri, and F. Priolo, Mater. Sci. Eng., B 114-115, 88 (2004).

${ }^{17}$ D. De Salvador, E. Napolitani, G. Bisognin, A. Carnera, E. Bruno, S. Mirabella, G. Impellizeri, and F. Priolo Mater. Sci. Eng., B 124-125, 88 (2005).

${ }^{18}$ O. Madelung, M. Schulz, eds., Numerical Data and Fundamental Relationships in Science and Technology, Landolt-Börnstein, New Series, Group III, Vol. 22, Part B (Springer, Berlin Heidelberg, 1989), p. 208 